\title{
Ein janusköpfiger Aufschwung: Beschäftigungsgewinne und Polarisierung
}

Ist der aktuelle Aufschwung am Arbeitsmarkt eine Folge struktureller Änderungen, die eine nachhaltige positive Entwicklung erwarten lassen, oder ein normales Konjunkturphänomen? Um diese Frage zu klären, vergleicht der Beitrag die Aufschwünge in den Jahren 1999/2000 sowie 2006/2007. Betrachtet werden makroökonomische Zusammenhänge zwischen Arbeitslosigkeit, Inflation und offenen Stellen, aber auch die Entwicklung der Langzeitarbeitslosigkeit, der Unterbeschäftigung und strukturelle Änderungen der Erwerbstätigkeit. Die Auswertung lässt vermuten, dass sich der Arbeitsmarkt nachhaltig positiv entwickelt. Es wird aber auch deutlich, dass vor allem Zugeständnisse der Arbeitnehmer und Arbeitslosen die Grundlagen des Aufschwungs gelegt haben.

\section{Einleitung}

Die Arbeitslosigkeit ist in den Jahren 2006 und 2007 deutlich gesunken. Kontrovers wird darüber diskutiert, welche Ursache diese günstige Entwicklung hat. Leisteten die Agenda 2010 und die Hartz-Reformen einen Beitrag? Oder befand sich die Wirtschaft in einem gewöhnlichen zyklischen Aufschwung? Dieser Frage geht der Beitrag nach. Dabei vergleichen wir die Entwicklung in den Jahren 2006 und 2007 mit dem Aufschwung der Jahre 1999 und 2000, also einem Aufschwung, der nach Ansicht der meisten Ökonomen einem normalen konjunkturellen Muster folgte. ${ }^{1}$

Eine rein konjunkturelle Änderung der Arbeitslosigkeit wird durch Schwankungen der gesamtwirtschaftlichen Nachfrage verursacht. Sie ist - nach der gängigen Lehrmeinung - kurzfristiger Natur, ändert die Arbeitslosigkeit also nicht dauerhaft. Das Niveau, um das die tatsächliche Arbeitslosigkeit im Konjunkturzyklus schwankt, wird als inflationsstabile Arbeitslosigkeit ${ }^{2}$ bezeichnet.

Nachhaltig sinkt die Arbeitslosigkeit nur, wenn die inflationsstabile Arbeitslosigkeit selbst sinkt. Ursache dafür kann zum einen eine Änderung in der Lohnund Preisbildung sein, die z. B. aus einer besseren Koordination der Lohnverhandlungen oder einem stärkeren Wettbewerb zwischen den Unternehmen resultiert.

Zum anderen kann die Ursache für eine nachhaltige Verringerung der Arbeitslosigkeit eine Verbesserung der sogenannten Matchingeffizienz sein. Von einer besseren Matchingeffizienz spricht man, wenn es leichter geworden ist, offene Stellen zu besetzen, weil Stellen- und Bewerberprofile hinsichtlich des Lohnes, der Arbeitsinhalte, -bedingungen und -orte besser zueinander passen. Es war ein wichtiges Ziel der HartzReformen, die Matchingeffizienz am Arbeitsmarkt zu verbessern. Darauf zielten so umstrittene Maßnahmen wie die Verschärfung von Zumutbarkeitsregeln oder die Verkürzung der Bezugsdauer des Arbeitslosengeldes I, aber auch die Förderung eines früheren Beginns der Arbeitssuche, z. B. durch die frühzeitige Meldung bei den Arbeitsagenturen und durch Job-to-JobVermittlung.

\section{2 \\ Aufschwung weicht vom üblichen Zyklus ab}

Um herauszufinden, welche Ursachen hinter einer Veränderung der Arbeitslosigkeit stecken, ist es hilfreich, zwei Zusammenhänge kombiniert zu betrachten (Blanchard 1990): die Phillipskurve (Abbildung 1) und die Beveridgekurve (Abbildung 2). Die Phillipskurve ist der Zusammenhang zwischen Inflation und Arbeitslosigkeit, die Beveridgekurve der zwischen offenen Stellen und Arbeitslosigkeit. Mit ihrer Hilfe lässt sich identifizieren, ob die Arbeitslosigkeit sich aufgrund einer Änderung in der Lohn- und Preisbildung, einer Änderung in der Matchingeffizienz oder aus konjunkturellen Gründen wandelt.

Im normalen Konjunkturzyklus zeigen beide Kurven ein typisches Muster: Eine geringere Arbeitslosigkeit ist verbunden mit höherer Inflation und einer höheren Zahl offener Stellen.
Genau dieses Muster war im Aufschwung um 1999 und 2000 zu beobachten. Ab 1999 stieg die gesamtwirtschaftliche Nachfrage. Damit wurde auch mehr Arbeit nachgefragt und die Zahl der offenen Stellen nahm zu. Größtenteils konnten sie auch besetzt werden, sodass die Arbeitslosigkeit sank. Aufgrund der sinkenden Arbeitslosigkeit konnten wieder höhere Löhne durchgesetzt werden als in den Jahren zuvor. Zum Teil wurden die höheren Löhne von den Unternehmen auf die Preise abgewälzt, sodass ab Mitte 2000 auch die Inflation zunahm.

Verändern sich offene Stellen, Inflationsrate und Arbeitslosigkeit in der be-

\footnotetext{
Der Beitrag nimmt keine exakte Abgrenzung der Aufschwungphasen vor. Verglichen werden die Jahre 1999/2000 mit 2006/2007. Veränderungen jahresdurchschnittlicher Größen werden gemäß dieser Abgrenzung von 1998 auf 2000 und von 2005 auf 2007 berechnet.

2 Sie wird manchmal auch strukturelle oder gleichgewichtige Arbeitslosigkeit genannt.
}

Hermann Gartner, Dr., Wissenschaftlicher Mitarbeiter im Institut für Arbeitsmarkt- und Berufsforschung (IAB), Nürnberg. Arbeitsschwerpunkte: Kurzfristige Arbeitsmarktentwicklung, Worker und job flows, Lohnentwicklung und Lohnungleichheit. e-mail: hermann.gartner@iab.de Sabine Klinger, Dr., Wissenschaftliche Mitarbeiterin im IAB. Arbeitsschwerpunkte: Kurzfristige Arbeitsmarktentwicklung, Wandel der Erwerbsformen, Politiksimulationen.

e-mail: sabine.klinger@iab.de 
schriebenen Weise, so bewegt sich die Volkswirtschaft auf der Phillips- und der Beveridgekurve. Solche Bewegungen auf den Kurven sind also ein Hinweis, dass Änderungen der Arbeitslosigkeit auf Änderungen der gesamtwirtschaftlichen Nachfrage zurückzuführen sind.

Ändert sich hingegen die inflationsstabile Arbeitslosigkeit, bricht die Volkswirtschaft aus der Bewegung auf den Kurven aus. Wenn sich die Phillipskurve nach links verschiebt, die Beveridgekurve aber stabil bleibt, dürften Änderungen in der Lohnund Preisbildung den Rückgang der inflationsstabilen Arbeitslosigkeit verursacht haben.

Wenn sich die Phillipskurve und die Beveridgekurve gemeinsam nach links verschieben, deutet das dagegen auf eine verbesserte Matchingeffizienz auf dem Arbeitsmarkt hin - wobei dann nicht auszuschließen ist, dass sich auch die Lohn- und Preisbildung geändert hat.

Einen ersten Eindruck darüber, ob sich im gegenwärtigen Aufschwung der Phillips- und der Beveridgekurvenzusammenhang verschoben haben, liefert zunächst die empirische Entwicklung der Kurven. Die empirische Phillipskurve zeigt: Die Arbeitslosigkeit ist deutlich stärker zurückgegangen als 1999 und 2000, während die Inflation etwas weniger angestiegen ist als damals. Der Anstieg der Inflation dürfte zudem kaum auf Lohndruck zurückzuführen sein, sondern auf die höhere Mehrwertsteuer. Ende 2007, bei einer wesentlich geringeren Arbeitslosigkeit als zum Tiefststand im Jahr 2001, scheint auch - anders als damals - die Zunahme der Inflation gestoppt zu sein. Zwar lässt die empirische Phillipskurve Interpretationsspielräume offen, dennoch lässt die stabilere Inflation bei geringerer Arbeitslosigkeit als 2001 eine Verschiebung der Phillipskurve nach links vermuten.

Deutlicher ist der ungewöhnliche Verlauf der Beveridgekurve. Zwar entwickelte sich die Zahl der Arbeitslosen und der offenen Stellen im Jahr 2006 noch nach dem konjunkturellen Muster : Die Arbeitslosigkeit sank, während die Zahl der offenen Stellen stieg. Doch im Jahr 2007 nahm auch die Zahl der offenen Stellen ab - während die Arbeitslosigkeit weiter sank. ${ }^{3}$ Offene Stellen können also häufiger besetzt werden als neue Stellen entstehen.

Ein klareres Bild, ob sich die Phillipsund die Beveridgekurve verschoben haben, zeigt die folgende Regressionsanalyse ana-

\section{Abb. 1: Phillipskurve, Januar 1998 bis Januar 2008}

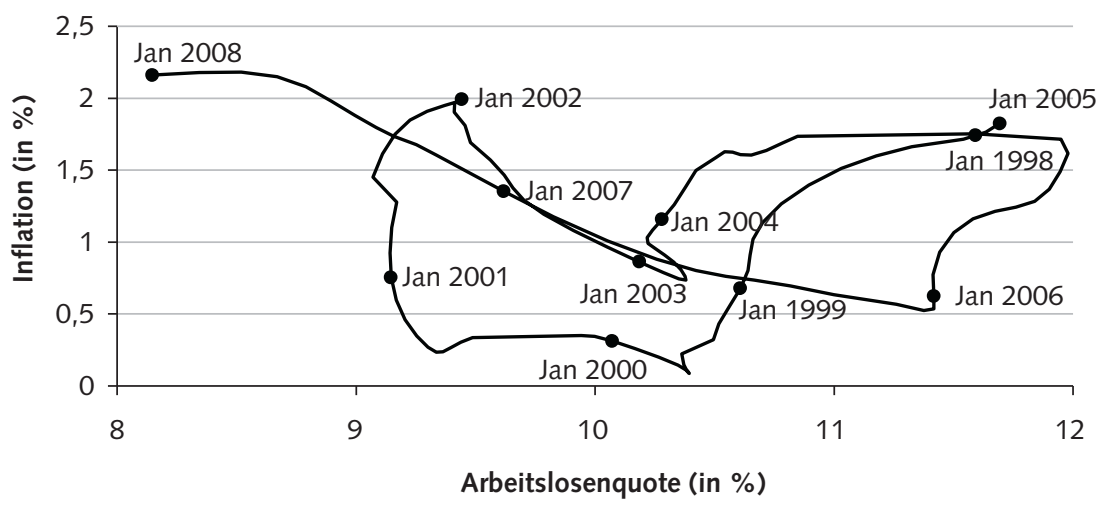

Saisonbereinigte Monatswerte, Inflation: Veränderung des Verbraucherpreisindex (ohne Energie und saisonabhängige Nahrungsmittel) gegenüber Vorjahr. Quelle: Destatis, Bundesagentur für Arbeit.

WSI MitTEILUNGeN

\section{Abb. 2: Beveridgekurve, Januar 2000 bis Januar 2008}

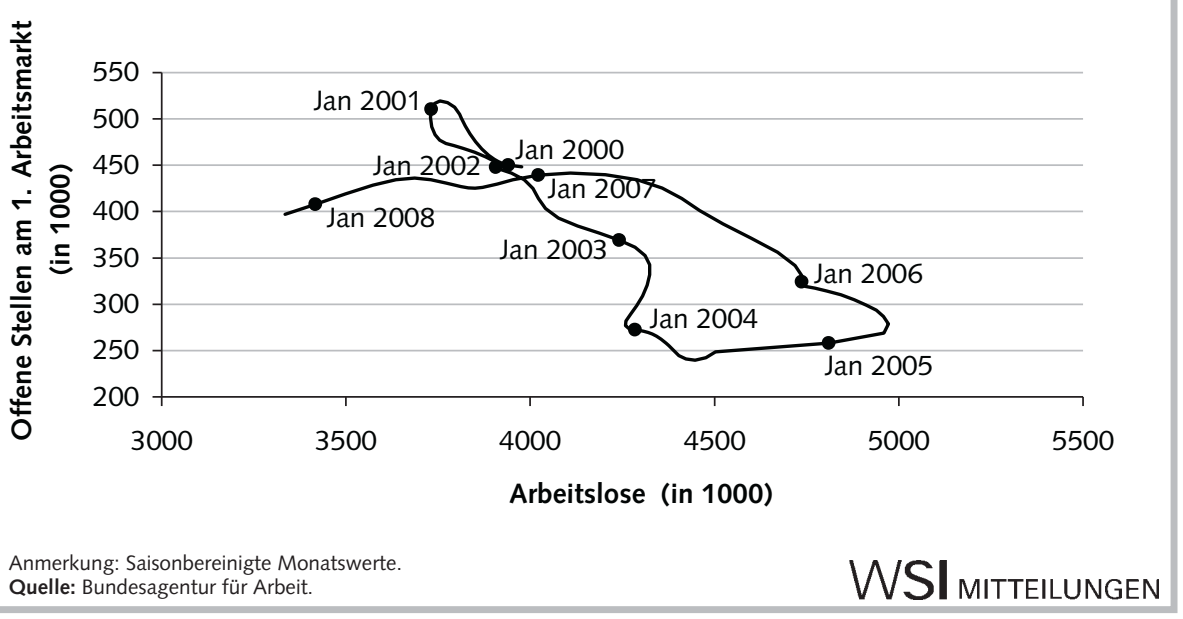

log zu Blanchard (1990). ${ }^{4}$ Ermittelt werden eine einfache Phillipskurve

$\log \left(\right.$ Arbeitslosenquote $\left._{\mathrm{t}}\right)=\alpha_{0}+\alpha_{1}$ Inflation $_{\mathrm{t}}$ $+\alpha_{2}$ Inflation $_{\mathrm{t}-1}+$ Residuum $_{\mathrm{t}}$

und eine Beveridgekurve

$\log \left(\right.$ Arbeitslosenquote $\left._{\mathrm{t}}\right)$

$=\beta_{0}+\beta_{1} \log \left(\right.$ Vakanzquote $_{\mathrm{t}}+$ Residuum $_{\mathrm{t}}$.

Geschätzt wurden die Kurven für die Monate von August 1999 bis April 2003, also für den Zeitraum zwischen den Tiefstwerten der Inflation im vergangenen Konjunkturzyklus. ${ }^{5}$ Für Januar 1993 bis Dezember 2007 wurden die Residuen, also die Abweichungen zwischen der geschätzten und der beobachteten Arbeitslosigkeit, berechnet (Abbildung 3). Sie zeigen eine Veränderung der Arbeitslosigkeit an, die nicht durch eine Veränderung der Inflation oder der
3 Die Grafik und die folgende Schätzung basieren auf den gemeldeten offenen Stellen. Stellen, die den Arbeitsagenturen nicht gemeldet werden, sind damit nicht erfasst. Kurz vor Redaktionsschluss standen aber auch Daten der IAB-Erhebung des gesamtwirtschaftlichen Stellenangebotes zur Verfügung. Diese Daten bestätigen den Rückgang der Vakanzen bei gleichzeitig sinkender Arbeitslosigkeit im Jahr 2007.

4 Eine ökonometrisch anspruchsvollere Strategie zur Identifikation der Ursachen der Schocks, die sich nun auf den Arbeitsmarkt auswirken, wäre die Schätzung struktureller vektorautoregressiver Modelle. Einfache Verfahren, wie das hier verwendete, können aber gute Anhaltspunkte liefern.

5 Verwendet wurden Monatsdaten. Inflation ist die Veränderung des Verbraucherpreisindex (ohne Energie und saisonabhängige Nahrungsmittel) gegenüber dem Vorjahresmonat. Die Vakanzrate gibt die gemeldeten offenen Stellen pro Erwerbsperson an. Die gemeldeten offenen Stellen am ersten Arbeitsmarkt (Abbildung 2) werden erst seit 2000 explizit ausgewiesen. Für die Schätzung wurden daher alle gemeldeten offenen Stellen genutzt, einschließlich ABM-Stellen und Arbeitsgelegenheiten. 
Abb. 3: Residuen der Phillips- und Beveridgekurve, 1993 bis 2007

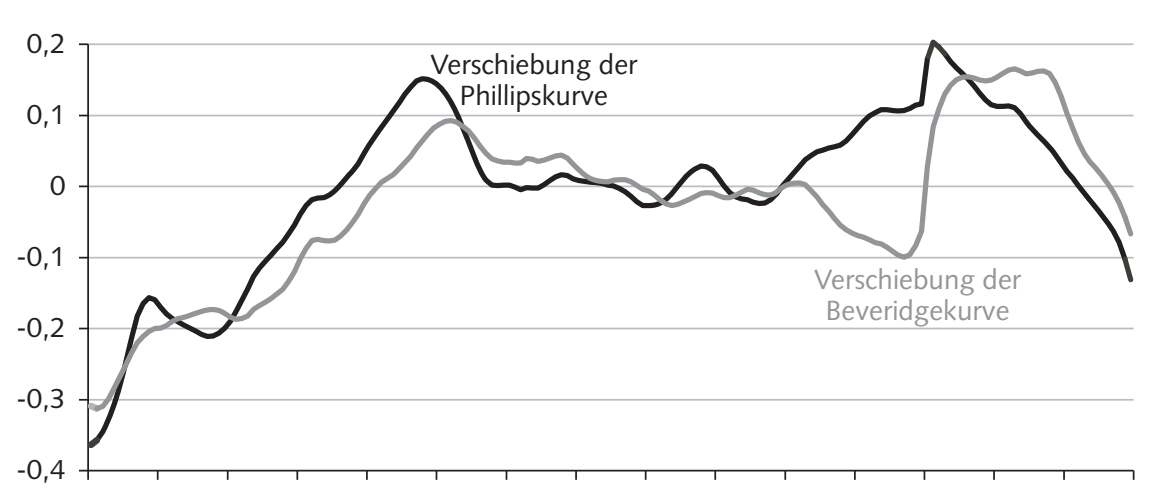

199319941995199619971998199920002001200220032004200520062007

Anmerkung: Arbeitslosigkeit als abhängige Größe.

Quelle: Berechnungen der Autoren auf Basis von Monatswerten.

WSI MITTEILUNGEN

offenen Stellen erklärt werden kann. Veränderungen der Residuen sind damit als Verschiebung der Kurven zu interpretieren.

In den Jahren 1996 und 1997 verschlechterte sich die Matchingeffizienz die Residuen beider Schätzungen bewegen sich parallel nach oben. Anfang 2005 bewegen sich beide Kurven erneut nach oben. Dies ist der Strukturbruch durch die HartzIV-Reform, mit der erwerbsfähige Sozialhilfeempfänger zur Arbeitslosigkeit hinzugezählt wurden. Bei der Beveridgekurve kommt hinzu, dass die Ein-Euro-Jobs die Zahl der offenen Stellen nach oben verschoben haben.

Im Lauf der Jahre 2005 bis 2007 aber verlaufen beide Residuen-Kurven gemeinsam nach unten. Dies bestätigt die Vermutung, dass der beobachtete Rückgang der Arbeitslosigkeit wenigstens zum Teil auf eine Verbesserung der Matchingeffizienz zurückzuführen und damit nachhaltig ist. ${ }^{6}$

Was steckt hinter der Verbesserung der Matchingeffizienz? Die Höhe der inflationsstabilen Arbeitslosigkeit hängt wesentlich von der verfestigten Sockelarbeitslosigkeit ab: dem Bestand an Langzeitarbeitslosen. Zum einen wird die Qualifikation eines Arbeitslosen mit zunehmender Dauer der Arbeitslosigkeit entwertet. Dadurch wird es immer schwerer, eine Arbeit zu finden, die so gut wie die alte Stelle entlohnt wird. Zum anderen wird eine Person, die länger arbeitslos ist, zunehmend entmutigt und sucht weniger intensiv nach Arbeit (Layard et al. 2005). Hier spielt auch ein Stigmaeffekt eine Rolle. Von einem Bewerber, der schon länger arbeitslos ist, kann ein Arbeitgeber die - möglicherweise falsche - Einschätzung haben, dass der Bewerber weniger produktiv oder motiviert

\section{Sockel der Langzeit- arbeitslosigkeit bröckelt}

Arbeitslose werden seit der Hartz-IV-Reform im Jahr 2005 in zwei Rechtskreisen SGB III und SGB II - registriert und betreut. Der Rechtskreis SGB III umfasst das System der Arbeitslosenversicherung mit der Bundesagentur für Arbeit (BA) als zentral Verantwortlicher. Die dort betreuten Arbeitslosen beziehen in der Regel Arbeitslosengeld I. Sie sind meist nur kurz arbeitslos und stehen dem Arbeitsmarkt deshalb näher. Im Rechtskreis des SGB II, dem System der Grundsicherung für erwerbsfähige Bedürftige, betreuen Kommunen und Arbeitsagenturen in Arbeitsgemeinschaften oder getrennter Trägerschaft oder aber zugelassene kommunale Träger die Arbeitslosen. Sie haben häufiger Vermittlungshemmnisse, sind schon länger ar- beitslos und erhalten - nach Prüfung der Bedürftigkeit - Arbeitslosengeld II. Personen, die länger als ein Jahr ununterbrochen als arbeitslos registriert sind, werden in der Statistik der Bundesagentur ${ }^{7}$ als „langzeitarbeitslos“ erfasst. ${ }^{8}$ Im Durchschnitt der Jahre 1998 bis 2007 waren 1,5 Mio. Personen langzeitarbeitslos, mehr als ein Drittel des durchschnittlichen Bestands von 4,1 Mio. Arbeitslosen.

Wie reagiert die Langzeitarbeitslosigkeit im Vergleich zur gesamten Arbeitslosigkeit gewöhnlich auf einen Aufschwung? Einerseits sind Langzeitarbeitslose meist geringer qualifiziert, ihnen fehlen soziale Netzwerke und sie haben geringere soziale Fähigkeiten (Kronauer/Vogel 1993). Um diese Beschäftigungshemmnisse zu kompensieren, dürfte eine Steigerung der Güternachfrage in einem Aufschwung allein nicht genügen. $\mathrm{Zu}$ erwarten wäre daher, dass im Aufschwung der Bestand an Langzeitarbeitslosen schwächer reagiert als der Bestand an Kurzzeitarbeitslosen.

Andererseits sind Arbeitsplätze im gering qualifizierten und gering entlohnten Bereich konjunkturreagibler: In einer Rezession werden gering qualifizierte Arbeitskräfte schneller entlassen, im Aufschwung aber auch schneller eingestellt. Von einem Aufschwung können daher Langzeitarbeitslose durchaus profitieren.

Diese beiden Effekte hielten sich in den Jahren 1999 und 2000 die Waage: Im Jah-
6 Als Robustheitstest wurden die Phillips- und die Beveridgekurve auch in umgekehrter Richtung geschätzt, also mit der Arbeitslosigkeit als erklärender statt als abhängiger Variable. Die Residuen dieser Schätzungen zeigen ein ähnliches Muster, sodass wir unsere Ergebnisse als stabil betrachten.

7 Daten zur Langzeitarbeitslosigkeit enthält das IT-Verfahren der BA ab Dezember 1997. Die Daten der zugelassenen kommunalen Träger sind darin ab 2005 nicht mehr enthalten. Zudem wurde der Stichtag für die Zählung der Arbeitslosen vom Monatsende auf die Monatsmitte vorverlegt. Vergleiche von Daten der BA vor und nach der Hartz-IV-Reform bedürfen deshalb besonderer Vorsicht, wenngleich die Verzerrungen gering sein dürften.

8 Die Registrierung wird aber z. B. durch eine Arbeitsgelegenheit oder Weiterbildungsmaßnahme oder durch Krankheit unterbrochen. Bei Personen, die danach in die Arbeitslosigkeit zurückwechseln, beginnt die Dauer der Arbeitslosigkeit von vorn. Sie sind in der Statistik also nicht mehr als langzeitarbeitslos erfasst. Erweitert man die Berechnung der Langzeitarbeitslosigkeit um die Teilnahme an Arbeitsgelegenheiten und Weiterbildungsmaßnahmen zeigt sich aber, dass beide Zeitreihen ähnlich verlaufen. Die hier getroffenen Aussagen sind damit robust gegenüber der Abgrenzung. 
resdurchschnitt sank die Arbeitslosigkeit in diesen beiden Jahren um 9,1\% oder 390.000, die der Langzeitarbeitslosen ebenfalls um 9,1 \% (150.000 Personen, Abbildung 4). Profitierten nach den Reformen am Arbeitsmarkt die Langzeitarbeitslosen nun stärker vom Aufschwung, sodass auch die Sockelarbeitslosigkeit abgebaut wird? Zunächst hatte die Hartz-IV-Reform zu Beginn der Jahre 2005 und 2006 zu statistischen Sprüngen geführt: Mit der Zusammenlegung von Arbeitslosen- und Sozialhilfe mussten sich vormals arbeitsfähige Sozialhilfeempfänger melden, um Arbeitslosengeld II beantragen zu können. Auch wenn sie vorher schon erwerbslos waren, wurden sie zunächst mit einer Dauer der Arbeitslosigkeit von null Monaten registriert. Der Anteil der Langzeitarbeitslosen an allen Arbeitslosen fiel dadurch. Blieben sie ein weiteres Jahr arbeitslos, wuchsen sie Anfang 2006 in die gemessene Langzeitarbeitslosigkeit hinein. Dies erklärt den kräftigen Sprung im Bestand der Langzeitarbeitslosigkeit. Als Folge stieg der Anteil der Langzeitarbeitslosen an allen Arbeitslosen auf $41 \%$.

In den Jahren 2006/2007 hat die Arbeitsnachfrage deutlich zugenommen aber auch ein demografisch bedingter Rückgang des Arbeitsangebots hat den Arbeitsmarkt entlastet. In der Folge sanken sowohl die Arbeitslosigkeit als auch die Langzeitarbeitslosigkeit wesentlich kräftiger als 1999/2000 (Abbildung 4): Im Jahresdurchschnitt verringerte sich die Arbeitslosigkeit von 2005 auf 2007 um 1,1 Mio. Personen oder $22,3 \%$ und die Langzeitarbeitslosigkeit um 200.000 Personen oder 12,7\%. Wie stark die Langzeitarbeitslosigkeit zurückgegangen ist, sieht man deutlicher, wenn man die Entwicklung seit ihrem Höchststand im März 2006 betrachtet, nachdem die ehemaligen Sozialhilfeempfänger in die erfasste Langzeitarbeitslosigkeit hineingewachsen waren. In weniger als zwei Jahren, bis Dezember 2007, ist die Langzeitarbeitslosigkeit um 530.000 Personen gefallen. Das ist doppelt so viel wie im Verlauf der vier Jahre von 1998 bis 2001.

Die Bestandsänderungen sind das Resultat großer Dynamik am Arbeitsmarkt: Permanent finden Zugänge in und Abgänge aus Arbeitslosigkeit statt. So verließen in den Jahren 1999/2000 im Schnitt monatlich 500.000 Personen (12,8 \% des Bestands im Vormonat) die Arbeitslosigkeit und rund 100.000 Personen $(6,7 \%$ des Bestands im Vormonat) die Langzeitarbeits-

\section{Abb. 4: Arbeitslosigkeit und Langzeitarbeitslosigkeit, Bestände 1998 bis 2007}

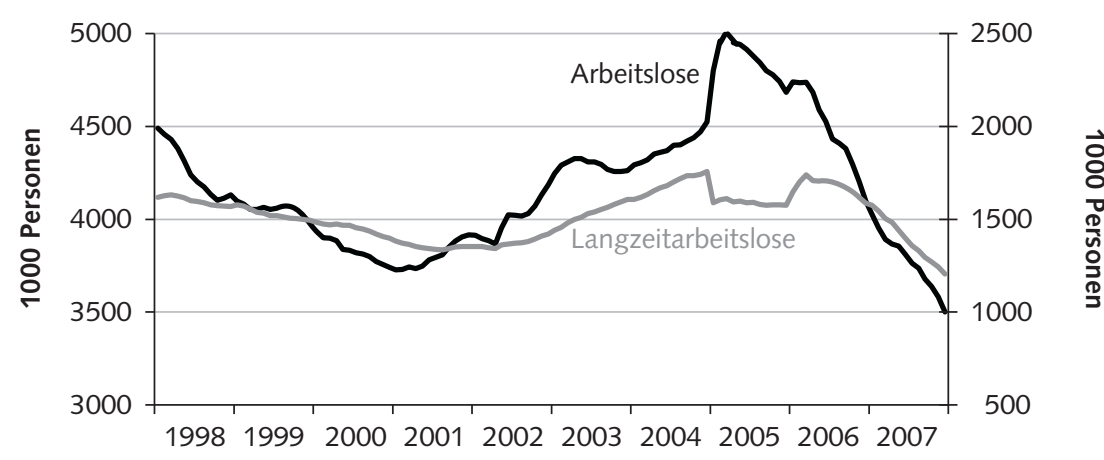

Anmerkung: Saisonbereinigte Monatswerte, ohne zugelassene Träger. Quelle: Bundesagentur für Arbeit, IAB.

WSI MitTEILUNGeN losigkeit (Abbildung 5). Schon ab dem Jahr 2003 stieg die Zahl der Abgänge wieder. Sie wurde damals aber deutlich überwogen von der Zahl der Zugänge, sodass der Bestand in jenem Jahr noch kräftig anstieg. Abgesehen vom Reformjahr 2005 wurde das hohe Niveau der Abgänge beibehalten und im Durchschnitt der Jahre 2006/2007 gab es monatlich 570.000 Abgänger (15,3\% des Bestands im Vormonat) aus Arbeitslosigkeit und 120.000 Abgänger (8,1 \% des Bestands im Vormonat) aus Langzeitarbeitslosigkeit.

Um die Auswirkung des aktuellen Aufschwungs zu beurteilen, ist entscheidend, wohin Langzeitarbeitslose gehen, wenn sie die Arbeitslosigkeit verlassen. Haben sie eine Beschäftigung bekommen, nehmen sie an Maßnahmen teil oder haben sie sich in die Nichterwerbstätigkeit zurückgezogen? Alle drei Abgangsziele haben gegenüber 1999/2000 an Bedeutung gewonnen.
Die Wechsel aus Langzeitarbeitslosigkeit in Beschäftigung auf dem ersten Arbeitsmarkt haben leicht zugenommen, von durchschnittlich 26.000 Personen (1,7\%) pro Monat in den Jahren 1999/2000 auf durchschnittlich 30.000 Personen (1,9\%) in den Jahren 2006/2007. Diese Aufwärtsbewegung hat - wie bei den Abgängen aus Arbeitslosigkeit allgemein - schon 2003 begonnen. Im Jahr 2007 sank die Zahl der Abgänge wieder. Diese Abnahme kann zum Teil mit dem gesunkenen Bestand an Langzeitarbeitslosen erklärt werden: Es wechseln weniger Personen aus der Langzeitarbeitslosigkeit in Beschäftigung, weil der Pool der infrage kommenden Personen kleiner geworden ist.

Kleiner wurde der Pool auch, weil Langzeitarbeitslose seit der Hartz-IV-Reform zunehmend mit (kürzeren) Weiterbildungs- und Trainingsmaßnahmen versorgt werden. 1999/2000 verließen durchschnittlich 9.000 Personen $(0,6 \%)$ pro Mo-

\section{Abb. 5: Abgänge aus (Langzeit-)Arbeitslosigkeit, 1998 bis 2007}

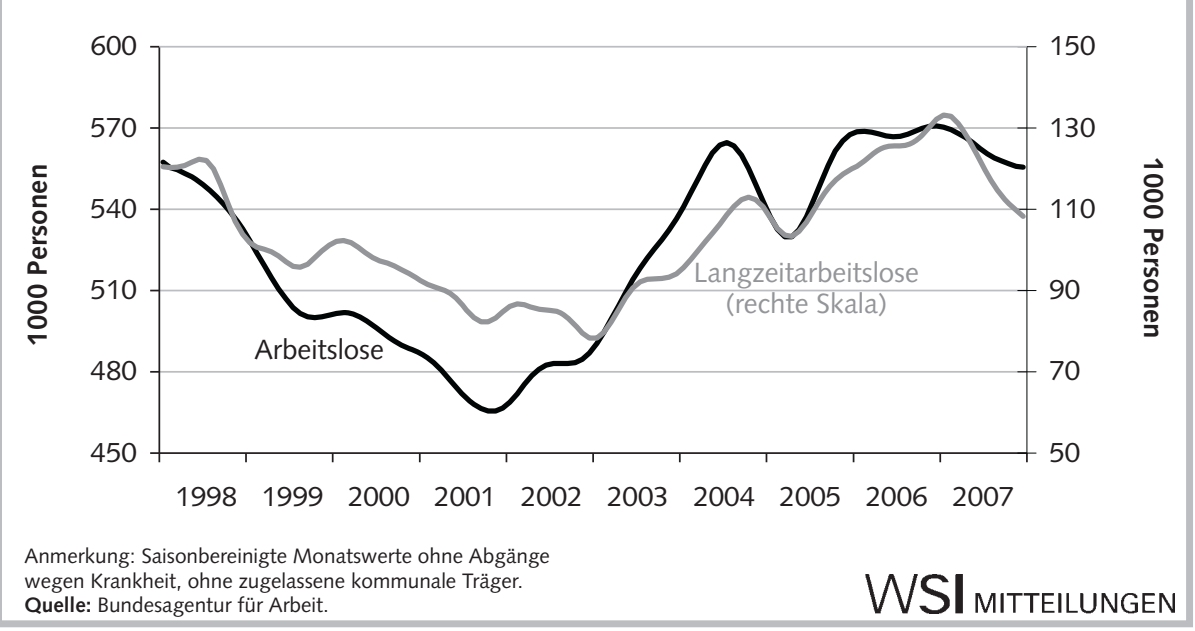


Abb. 6: Wachstumsbeiträge zur Unterbeschäftigung, 1992 bis 2007

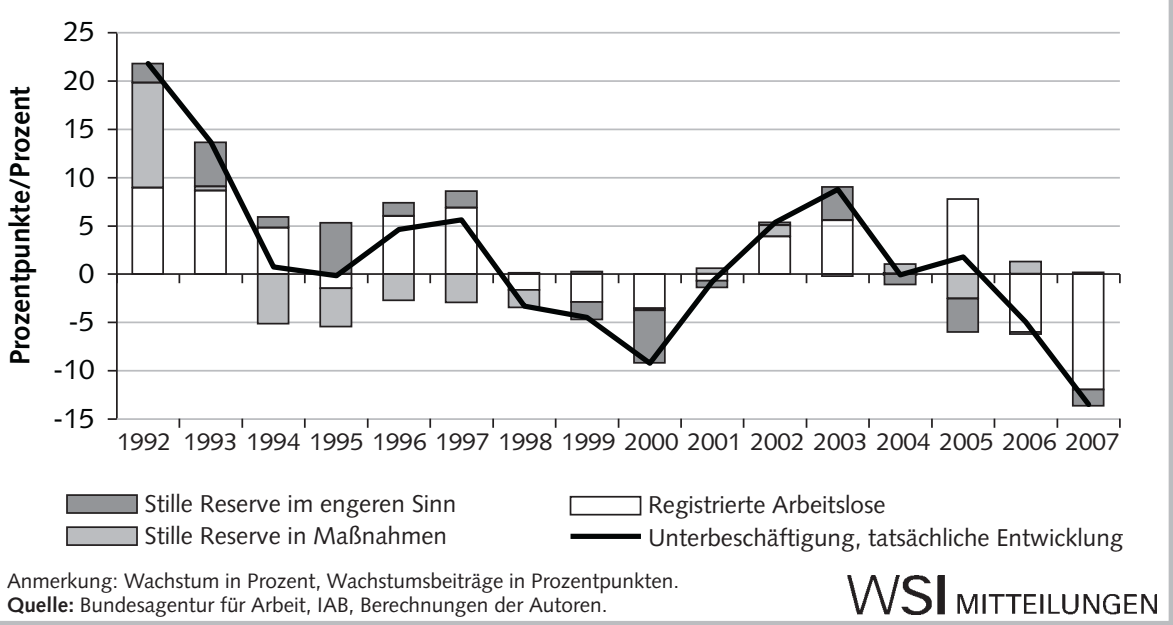

nat die Langzeitarbeitslosigkeit, um eine solche Maßnahme zu beginnen; 2006/2007 waren es durchschnittlich 19.000 Personen $(1,2 \%)$ pro Monat. Zugenommen haben auch die Abgänge in Beschäftigung schaffende Maßnahmen auf dem zweiten Arbeitsmarkt, von 10.000 Personen $(0,7 \%)$ pro Monat 1999/2000 auf 18.000 Personen $(1,2 \%)$ pro Monat in den Jahren 2006/ 2007. Diese Maßnahmen beeinflussen die Beschäftigungsperspektiven von Arbeitslosen unterschiedlich.

Die Zahl der Übergänge aus Langzeitarbeitslosigkeit in Nichterwerbstätigkeit (ohne Krankheit) hat sich nur wenig erhöht: von 54.000 Personen (3,6 \%) in den Jahren 1999/2000 auf 56.000 Personen $(3,7 \%)$ pro Monat in $2006 / 2007$. Dazu zählen Personen, die in den Ruhestand gehen, aber auch Personen, die sich nicht arbeitslos melden, da sie ihre Arbeitsmarktchancen als schlecht einschätzen. Sie sind entmutigt und suchen erst bei höherem Beschäftigungsstand aktiv nach einer Stelle. Sie bilden die sogenannte Stille Reserve im engeren Sinn und damit eine Komponente der Unterbeschäftigung.

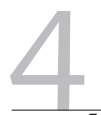

\section{Auch die Unterbeschäfti- gung geht zurück}

Die Unterbeschäftigung erfasst die Erwerbslosigkeit breiter als die registrierte Arbeitslosigkeit, weil sie zusätzlich die Stille Reserve im engeren Sinn und die Stille Reserve in Maßnahmen ${ }^{9}$ beinhaltet. Wenn analysiert wird, ob die inflationsstabile Arbeitslosigkeit sinkt, sollte die Diskussion daher auch auf die Unterbeschäftigung ausgedehnt werden.

Im Jahr 1998 führte die beginnende wirtschaftliche Erholung erstmals seit der Wiedervereinigung dazu, dass die Unterbeschäftigung sank, im Aufschwung 1999/ 2000 um insgesamt 13,3\% oder 834.000 Personen. Allein im Jahr 2000 ist die Unterbeschäftigung um 9,2\% gesunken (Linie in Abbildung 6). 3,5 Prozentpunkte davon sind auf die Abnahme der registrierten Arbeitslosigkeit zurückzuführen (Wachstumsbeitrag, gestapelte Balken in Abbildung 6). Es fanden aber auch Personen aus der Stillen Reserve im engeren Sinn Arbeit, sie trugen weitere 5,5 Prozentpunkte zum Rückgang der Unterbeschäftigung bei. Arbeitsmarktpolitische Maßnahmen entlasteten die Arbeitslosigkeit kaum; sie senkten die Veränderungsrate der Unterbeschäftigung nur um 0,2 Prozentpunkte.

Im ersten Jahr der Hartz-IV-Reform, 2005, stieg die Zahl der registrierten Arbeitslosen deutlich. Der Anstieg ist aber im Wesentlichen durch eine Umschichtung innerhalb der Unterbeschäftigung entstanden: Erwerbsfähige Sozialhilfebezieher, die vor der Reform in der Stillen Reserve im engeren Sinn erfasst waren, wurden nun als arbeitslos registriert. Verdeckte Arbeitslosigkeit wurde damit offen gelegt und die Stille Reserve im engeren Sinn sank. Aber auch die Stille Reserve in Maßnahmen nahm 2005 ab, wohl auch, weil die organisatorische Struktur der BA umgestaltet und der Einsatz von Instrumenten stärker an ihre Wirksamkeit gekoppelt wurde. Im Ergebnis nahm die Unterbeschäftigung nur um $1,9 \%$ zu.

Im Aufschwung 2006 und 2007 verringerte sich die registrierte Arbeitslosigkeit kräftig, während sich an der Stillen Reserve vergleichsweise wenig änderte. Die Unterbeschäftigung betrug 2007 5,1 Mio. Personen - ein derart niedriger Stand war zuletzt 1992 erreicht worden. Der überwiegende Teil der Personen, die die Unterbeschäftigung verlassen haben, sind in Beschäftigung gewechselt. Auch bei dieser breiteren Definition von Erwerbslosigkeit hat sich also der Arbeitsmarkt im aktuellen Aufschwung günstiger entwickelt als 1999 und 2000.

\section{Der Aufschwung ist beschäftigungsintensiver}

Die sozialversicherungspflichtige Beschäftigung nahm im Jahr 2007 stark zu, sie erreichte aber mit durchschnittlich 26,9 Mio. Personen längst nicht den Bestand des Jahres 2000 von 27,9 Mio. Personen. Wie ist das kompatibel mit der Vermutung, dass die inflationsstabile Arbeitslosigkeit derzeit sinkt? Die Erwartung, dass das frühere Niveau der sozialversicherungspflichtigen Beschäftigung wieder erreicht würde, lässt einen wichtigen Aspekt außer Acht: Die Beschäftigungsentwicklung ist das Ergebnis sowohl kurzfristiger Bewegungen als auch langfristiger struktureller Änderungen.

So hat sich die Struktur der Beschäftigung seit der Wiedervereinigung gravierend geändert (Abbildung 7): Die Zahl der Vollzeitstellen nahm beinahe kontinuierlich ab. Bis zum Jahr 2007 fiel sie auf weniger als $80 \%$ des Bestands von 1991. Der Aufschwung 1999/2000 konnte den Abbau gerade bremsen - erst 2007 ist erstmals eine leichte Aufwärtsbewegung zu verzeichnen. Dagegen gab es 2007 gegenüber 1991 zweimal so viele Teilzeitbeschäftigte und zweieinhalbmal so viele ausschließlich geringfügig Beschäftigte ${ }^{10}$.

\footnotetext{
9 Zur Stillen Reserve in Maßnahmen zählen die oben erwähnten Teilnehmer an Weiterbildungs- und Trainingsmaßnahmen, aber auch Personen über 58 Jahren, die die §§ 428 SGB III und 65 SGB II in Anspruch nehmen und Leistungen beziehen, ohne dass sie dem Arbeitsmarkt noch zur Verfügung stehen müssen. Diese Regelung lief zum 31.12. 2007 aus, und der betreffende Teilbestand der Stillen Reserve in Maßnahmen schmilzt nun ab.

10 Die ausschließlich geringfügige Beschäftigung schließt Arbeitslose mit einem geringfügigen $\mathrm{Ne}-$ benjob sowie Personen mit Ein-Euro-Jobs ein.
} 
Die starke Zunahme der Teilzeit- und der geringfügigen Beschäftigung hat institutionelle und ökonomische Ursachen. So setzen das Teilzeit- und Befristungsgesetz, das Altersteilzeitgesetz und das Gesetz über die geringfügige Beschäftigung Anreize für Arbeitnehmer und Arbeitgeber, solche Erwerbsformen zu nutzen. Zudem sehen sich Unternehmen durch die steigende Konkurrenz auf den Gütermärkten auch gezwungen, verstärkt auf flexible und kostengünstigere Erwerbsformen wie Teilzeit- und geringfügige Beschäftigung zu setzen (Wanger 2006; OECD 1999). In der Zunahme der geringfügigen Beschäftigung spiegeln sich aber zum Teil auch rein statistische Effekte. ${ }^{11}$

Der langfristige Wandel der Beschäftigungsstruktur ist zu berücksichtigen, wenn der Aufschwung 2006/2007 beurteilt wird. Tabelle 1 zeigt die Bestandsänderungen des Arbeitsangebots und der Arbeitsnachfrage, die 1999/2000 und 2006/2007 eintraten (vgl. hierzu auch Bach/Spitznagel 2007). Im Aufschwung 1999/2000 waren knapp 1,2 Mio. Arbeitnehmer mehr als zuvor beschäftigt, 2006/2007 nur 800.000. War der Aufschwung damals beschäftigungsintensiver? Die Antwort auf diese Frage hängt vom Maß für Beschäftigungsintensität $a b$. Legt man die Zahl der hinzugekommenen Arbeitnehmer oder Erwerbstätigen zugrunde, wurde in den Jahren 2006/2007 weniger zusätzliche Arbeit eingesetzt. Basiert die Einschätzung hingegen auf dem Arbeitsvolumen, der von allen Erwerbstätigen erbrachten Stundenzahl, wurde in den Jahren 2006/2007 mehr zusätzliche Arbeit eingesetzt als 1999/2000. Zu erklären ist der Unterschied mit der unterschiedlichen Entwicklung der Beschäftigungsstruktur in den jeweiligen Aufschwungjahren. 98 \% der zusätzlichen Beschäftigung von 1999/2000 war Teilzeitbeschäftigung - 2006/2007 waren es hingegen $74 \%$. Am Anstieg der Teilzeitbeschäftigung machte 1999/2000 die geringfügige Beschäftigung mit $54 \%$ mehr als die Hälfte aus - und damit auch etwa die Hälfte des gesamten Beschäftigungszuwachses. 2006/2007 machte die geringfügige Beschäftigung nur $21 \%$ des Anstiegs der Teilzeitbeschäftigung aus und nur $14 \%$ des gesamten Beschäftigungszuwachses.

Auch mit der Eingrenzung auf die sozialversicherungspflichtige Beschäftigung ist der Aufschwung 2006/2007 etwas beschäftigungsintensiver. Es wurden 50.000 Stellen mehr als 1999/2000 geschaffen, was ausschließlich auf den um 70.000 stärkeren

\section{Abb. 7: Entwicklung der Erwerbstätigkeit nach Personengruppen, 1991 bis 2007}

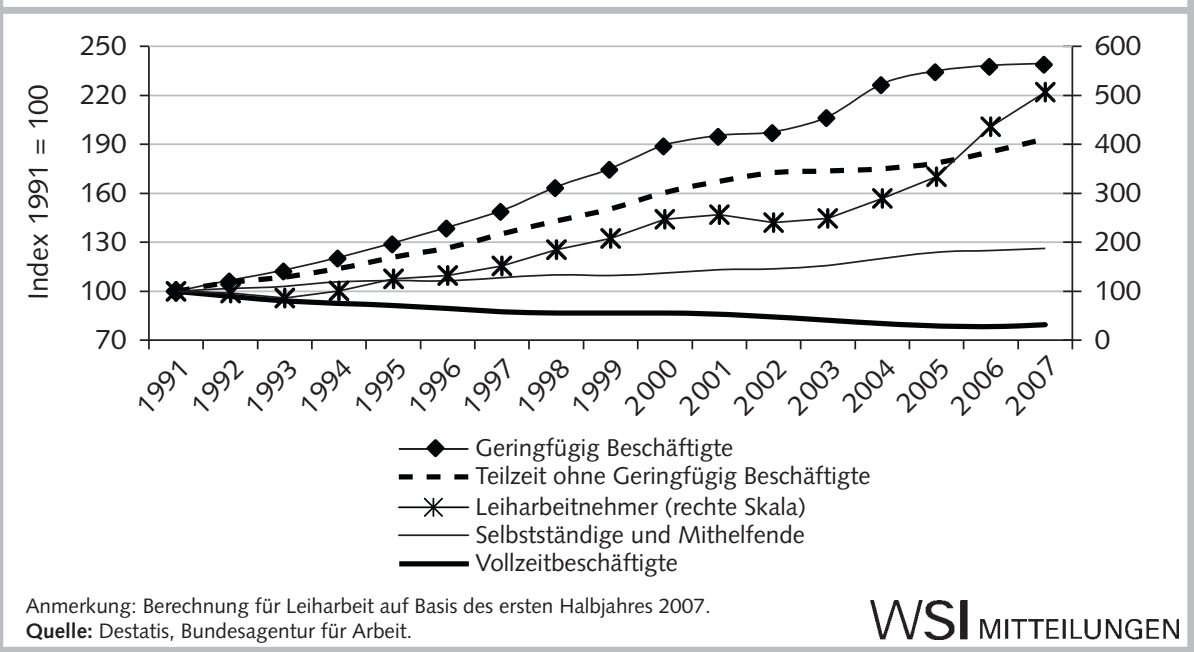

Tabelle 1: Konjunkturaufschwung im Vergleich, 1999/2000 und
2006/2007

\begin{tabular}{|c|c|c|c|c|}
\hline & \multicolumn{4}{|c|}{ Bestandsänderung } \\
\hline & \multicolumn{2}{|c|}{1998 auf 2000} & \multicolumn{2}{|c|}{2005 auf 2007} \\
\hline & in $\%$ & in 1000 & in $\%$ & in 1000 \\
\hline \multicolumn{5}{|l|}{ Arbeitskräfteangebot } \\
\hline Erwerbspersonenpotenzial & $+0,9$ & +379 & $-0,2$ & -99 \\
\hline \multicolumn{5}{|l|}{ Arbeitskräftenachfrage } \\
\hline Bruttoinlandsprodukt, preisbereinigt & $+5,3$ & & $+5,4$ & \\
\hline Stundenproduktivität & $+4,1$ & & $+3,2$ & \\
\hline Arbeitsvolumen & $+1,2$ & & $+2,2$ & \\
\hline Tatsächliche Arbeitszeit & $-2,0$ & & $-0,1$ & \\
\hline Erwerbstätige & $+3,3$ & +1.233 & $+2,3$ & +890 \\
\hline Selbstständige und Mithelfende & $+1,3$ & +50 & $+2,1$ & +90 \\
\hline Beschäftigte Arbeitnehmer & $+3,5$ & +1.183 & $+2,3$ & +800 \\
\hline Vollzeit & $+0,1$ & +22 & $+0,9$ & +211 \\
\hline Teilzeit & $+13,8$ & +1.161 & $+5,2$ & +589 \\
\hline Herkömmliche Teilzeit & $+12,2$ & +533 & $+8,5$ & +463 \\
\hline Geringfügig Beschäftigte (IAB-Schätzung) & $+15,5$ & +628 & $+2,2$ & +126 \\
\hline Arbeitnehmer 1. Arbeitsmarkt & $+3,3$ & +1.106 & $+2,4$ & +831 \\
\hline Arbeitnehmer 2. Arbeitsmarkt & $+20,4$ & +77 & $-7,5$ & -31 \\
\hline Sozialversicherungspflichtig Beschäftigte & $+2,4$ & +645 & $+2,7$ & +696 \\
\hline Vollzeit & $+1,1$ & +254 & $+1,5$ & +326 \\
\hline Teilzeit & $+11,2$ & +391 & $+8,5$ & +371 \\
\hline Zeitarbeit* & $+33,3$ & +82 & $+64,0$ & +284 \\
\hline
\end{tabular}

Anstieg der sozialversicherungspflichtigen Vollzeitstellen zurückzuführen ist. ${ }^{12}$

Dass in den Jahren 1999/2000 die Erwerbstätigkeit stärker anstieg als 2006/ 2007, aber die Arbeitslosigkeit weniger zurückging, macht deutlich, dass für die Beurteilung des Aufschwungs auch das Erwerbspersonenpotenzial zu berücksichtigen ist. 1999/2000 hat es den Arbeitsmarkt mit 380.000 zusätzlichen Personen belastet. Dies hängt vor allem mit der Entwicklung der geringfügigen Beschäftigung zusammen: Der hohe Anteil geringfügiger Beschäftigung beim Zuwachs der Erwerbs-
11 Bei der geringfügigen Beschäftigung wurde die Meldepflicht 1999 verschärft. Zudem führte die Anhebung der Einkommensgrenze 2003 auf $400 €$ dazu, dass früher sozialversicherungspflichtige Beschäftigungsverhältnisse nun unter die Geringügigkeitsgrenze fielen.

12 In Tabelle 1 fallen 1999/2000 die Veränderungen der beschäftigten Arbeitnehmer in Vollzeit (+22.000) und der sozialversicherungspflichtig Beschäftigten in Vollzeit (+254.000) weit auseinander. Die Zahl der Beamten sowie der nicht sozialversicherungspflichtigen Arbeiter und Angestellten in Vollzeit sank in diesen beiden Jahren kräftig und kompensierte das Plus bei den Vollzeitstellen mit Sozialversicherungspflicht fast vollständig. 
tätigkeit 1999/2000 ist auch Ausdruck einer höheren Erwerbsneigung. Hauptsächlich Schüler, Studenten, Rentner oder Ehefrauen begannen eine geringfügige Beschäftigung. Sie waren vorher nicht arbeitslos registriert und nicht im Erwerbspersonenpotenzial erfasst (BA 2001, S. 47; Fertig et al. 2005). Wenn geringfügige Beschäftigung entsteht, vermindert dies die Arbeitslosigkeit also wesentlich schwächer, als wenn sozialversicherungspflichtige Beschäftigung entsteht. ${ }^{13}$ Der Anstieg der geringfügigen Beschäftigung und des Erwerbspersonenpotenzials sind demnach miteinander verbunden. Wenn das Erwerbspersonenpotenzial 1999/2000 weniger gewachsen wäre, wäre deswegen nicht automatisch die Arbeitslosigkeit stärker gesunken.

Einzuschränken wäre diese Argumentation, wenn sozialversicherungspflichtige Beschäftigung in hohem Maß durch geringfügige Beschäftigung ersetzt worden wäre. Es ist aber nicht anzunehmen, dass die Neigung zur Substitution 1999/2000 höher geworden wäre. ${ }^{14}$ Im Gegenteil: Durch die Reform 1999 belegte der Gesetzgeber geringfügige Beschäftigungsverhältnisse mit höheren Abgaben, verschärfte die Meldepflicht und schrieb die Geringfügigkeitsgrenze (statt bisher jährlicher Dynamisierung) für mehrere Jahre bei $630 \mathrm{DM}$ $(325 €)$ fest. Dadurch verlor geringfügige Beschäftigung gegenüber sozialversicherungspflichtiger eher an Attraktivität (Rudolph 1999).

Anders als im vorigen Aufschwung ging das Arbeitsangebot in den Jahren 2006/ 2007 erstmalig zurück (um 100.000 Personen) und leistete einen Beitrag zum Rückgang der Unterbeschäftigung. Insgesamt sank die Unterbeschäftigung aber um 1,1 Mio. Personen, was vor allem durch die gestiegene Erwerbstätigkeit begründet ist.

Zur Zunahme der Erwerbstätigkeit dürften mehrere Faktoren beigetragen haben. Die wesentlichen Lasten mussten aber die Arbeitnehmer schultern. Zum einen hat die moderate Lohnentwicklung der vergangenen Jahre die Wettbewerbsfähigkeit der Unternehmen vergrößert und so die Arbeitsnachfrage erhöht (Heilemann/Ulrich 2008). Zum anderen dürfte auch die Konzessionsbereitschaft arbeitsloser Bewerber gestiegen sein (Kettner/Rebien 2007). So gaben Betriebe in einer repräsentativen Befragung an, dass arbeitslose Bewerber seit der Hartz-IV-Reform eher bereit waren, auch Stellen unter ihrem Qualifikationsniveau, zu niedrigeren Löhnen oder schlechteren Arbeitsbedingungen (Schichtdienste, Arbeitszeiten, Anfahrtswege) anzunehmen. Insbesondere die Betriebe, die solche Zugeständnisse der Bewerber festgestellt hatten, konnten auch ihre Stellen leichter besetzen. Die höhere Konzessionsbereitschaft und Flexibilität dürfte einen Beitrag dazu geleistet haben, dass der Nachschub in die Langzeitarbeitslosigkeit zurückgeht. Gerade in der Phase, bevor ein Arbeitslosengeld-I-Empfänger auf Arbeitslosengeld II zurückfällt, dürfte die Konzessionsbereitschaft stark zugenommen haben.

Ein weiterer Hinweis auf die gestiegenen Flexibilitätsanforderungen an die Arbeitsuchenden findet sich, wenn betrachtet wird, wo überproportional Vollzeitstellen entstanden sind: im Bereich der Leiharbeit (Abbildung 7). Ein wesentlicher Grund für die Beschäftigung von Leiharbeitern liegt in der hohen Flexibilität, mit der Produktionslücken geschlossen und kurzfristige Engpässe zu geringen Anpassungskosten überbrückt werden können (Buscher 2007).

Die Beschäftigung in der Leiharbeitsbranche ist in Deutschland kontinuierlich gewachsen. In den Jahren 2006/2007 verzeichnete sie mit $64 \%$ ihren kräftigsten Zuwachs innerhalb von zwei Jahren überhaupt (das zweite Halbjahr 2007 wurde geschätzt). Sie leitete den Aufschwung am Arbeitsmarkt - gemeinsam mit dem Gastgewerbe und der Branche Verkehr und Nachrichtenübermittlung - schon ein, als die übrige sozialversicherungspflichtige $\mathrm{Be}$ schäftigung noch kräftig abgebaut wurde. Im Verlauf des Aufschwungs hat sich der Anteil der Leiharbeit am gesamten Beschäftigungsaufbau deutlich verringert, ist aber im Vergleich zur früheren Aufschwungphase nach wie vor hoch.

Eine Bewertung der zunehmenden Leiharbeit muss gemischt ausfallen. Leiharbeit kann positiv wirken, sofern mehr Menschen die Chance erhalten, sich überhaupt in einem Betrieb zu bewähren, soziale Kontakte wahrzunehmen und sich in die Erwerbsgesellschaft zu integrieren. Dies betrifft gerade erwerbslose Menschen. Im ersten Halbjahr 2007 waren zwei Drittel der Personen, die eine Leiharbeit aufnahmen, zuvor ohne Beschäftigung gewesen. Bei insgesamt $13 \%$ der neuen Leiharbeitnehmer lag die letzte Beschäftigung länger als ein Jahr zurück, und für weitere 7,5 \% war es die erste Beschäftigung überhaupt (Quelle: BA). Leiharbeit kann also gerade Personengruppen den Markteinstieg bieten, die sonst schwer vermittelbar sind. Promberger (2006) und CIETT (2000) zeigen einen „Klebeeffekt" der Leiharbeitnehmer in der Entleihfirma von 12 bis $15 \%$ bzw. in einer sozialversicherungspflichtigen Beschäftigung überhaupt von bis zu $30 \%$.

Andererseits ist die Beschäftigung in den Entleihbetrieben oft instabil. Die Verweildauer betrug $2003 \mathrm{im}$ Median 2,1 Monate und Kettenverträge waren verbreitet (Antoni/Jahn 2006). Zudem besteht das Risiko, dass Arbeitnehmerschutzregeln und Lohnabsprachen ausgehebelt oder reguläre Stellen verdrängt werden. Entleihfirmen investieren bei der kurzen Beschäftigungszeit kaum in die Qualifikation der Leiharbeitnehmer (zu Negativeffekten der Leiharbeit vgl. u.a. Autor/Houseman 2005).

Die Verringerung der inflationsstabilen Arbeitslosigkeit ist also mit einer Ausdehnung der prekären Beschäftigung verbunden - der Preis der positiven Entwicklung auf dem Arbeitsmarkt.

\section{Zusammenfassung und Schlussfolgerungen}

Erstmals seit den 1970er Jahren liegt im Aufschwung 2006/2007 die Sockelarbeitslosigkeit nicht höher als im vorangehenden Aufschwung, sondern niedriger. Bislang hatte die Arbeitslosigkeit von Aufschwung zu Aufschwung allein im alten Bundesgebiet um etwa 720.000 Personen zugenommen. Die Entwicklungen der Phillipskurve und der Beveridgekurve lassen hoffen, dass diese Änderung nachhaltig ist. Auch die Entwicklungen der Langzeitarbeitslosigkeit und der Unterbeschäftigung zeigen, dass der Arbeitsmarkt in Bewegung gekommen ist. Der verfestigte Kern der Arbeitslosigkeit bröckelt.

\footnotetext{
13 Zudem war 1999/2000 die Begrenzung des Stundenvolumens einer geringfügigen Beschäftigung bei 15 Stunden pro Woche noch in Kraft. Eine arbeitslose Person, die eine geringfügige Beschäftigung aufnahm, verlor dadurch nicht den Status der Arbeitslosigkeit.

14 Die Literatur beschränkt sich im Wesentlichen auf Substitutionseffekte nach der Reform im Jahr 2003, die schon wegen der Anhebung der Geringfügigkeitsgrenze auf $400 €$ zu einer "Umwidmung " sozialversicherungspflichtiger in geringfügige Beschäftigung geführt hat (Rudolph 2003). Indizien für eine Substitution darüber hinaus werden in begrenztem Umfang gesehen (BA 2004; Fertig et al. 2005)
} 
Eine Analyse, welche Beschäftigung entstanden ist, macht aber deutlich: Der Aufschwung vollzog sich im Wesentlichen auf den Schultern der Arbeitnehmer. Die Deregulierung der Leiharbeit und die Erleichterung geringfügiger und befristeter Verhältnisse haben zur Ausweitung prekärer Beschäftigung geführt. Zudem haben Arbeitnehmer über mehrere Jahre eine moderate Lohnentwicklung hingenommen. Auch der Druck auf Arbeitsuchende wurde erhöht.

Die zunehmende Beschäftigung musste womöglich mit zunehmender Polarisierung erkauft werden. Eine Umkehr in der Arbeitsmarkt- und Lohnpolitik - wie zum Teil schon geschehen - birgt dann die Gefahr, dass ein weiterer Abbau der Arbeitslosigkeit erschwert wird. Die Politik steht al- so vor der Aufgabe, einerseits den Forderungen nach einem Ende der Zumutungen entgegenzukommen und andererseits den Beschäftigungsaufbau nicht zu gefährden. Beides ist auch erreichbar. Das zeigen nicht zuletzt Länder wie Dänemark oder die Niederlande mit ihren Flexicurity-Konzepten, die sowohl Sicherheit als auch Flexibilität am Arbeitsmarkt gewährleisten.

So lässt sich eine hohe Matchingeffizienz nicht nur durch Fordern erreichen, sondern auch durch eine verstärkte Förderung der Arbeitsuchenden - möglichst bevor sie Teil des verfestigten Kerns der Langzeitarbeitslosen werden.

Ein höheres Risiko, langzeitarbeitslos zu werden, tragen Geringqualifizierte. Ihre Beschäftigungschancen können verbessert werden, wenn im unteren Lohnsegment der große Unterschied zwischen Bruttound Nettolohn verringert wird. Hierzu wäre erforderlich, das Arbeitseinkommen von der Finanzierung des Sozialstaates stärker zu entlasten und andere Einkommensquellen zu belasten.

Auch gibt es Möglichkeiten, die hohen Anreize für prekäre Beschäftigung zu vermindern und stattdessen jene für abgesicherte Vollzeitbeschäftigung zu erhöhen (z. B. Bofinger et al. 2006).

Weil die sich ausweitende Teilzeit- und prekäre Beschäftigung oft mit weniger Einkommen, Stabilität und Qualifizierungsmöglichkeiten im Betrieb verbunden sind, sollten zudem Maßnahmen überdacht werden, um die Aufstiegsmobilität zu fördern, etwa durch berufsbegleitende Weiterbildung.

\section{LITERATUR}

Antoni, M./Jahn, E. J. (2006): Arbeitnehmerüberlassung: Boomende Branche mit hoher Fluktuation, IAB-Kurzbericht 14, Nürnberg Autor, D. H./Houseman, S. N. (2005): Do temporary help jobs improve labor market outcomes for low-skilled workers? Evidence from random assignments, Upjohn Institute Staff Working Paper 124, Michigan Bach, H.-U./Spitznagel, E. (2007): Aufschwung am Arbeitsmarkt - Die Entwicklungsmuster wandeln sich, IAB-Forum 2, S. 36-43

Blanchard, O. (1990): Two Tools for Analyzing Unemployment, in: Nerlove, M. (Hrsg.): Issues in Contemporary Economics, S. 102-107 Bofinger, P./Dietz, M./Genders, S./Walwei, U. (2006): Vorrang für das reguläre Arbeitsverhältnis: Ein Konzept für Existenz sichernde Beschäftigung im Niedriglohnbereich, Gutachten für das Sächsische Ministerium für Wirtschaft und Arbeit (SMWA)

Bundesagentur für Arbeit (BA) (2001): Arbeitmarkt 2000, Amtliche

Nachrichten der Bundesanstalt für Arbeit 49, Nürnberg

Bundesagentur für Arbeit (BA) (2004): Mini- und Midijobs in Deutschland, Sonderbericht, Nürnberg

Buscher, H. S. (2007): Leiharbeit - ein Schmuddelkind des deutschen Arbeitsmarktes?, in: Wirtschaft im Wandel 2, S. 47-53

CIETT (2000): Orchestrating the evaluation of private employment agencies towards a stronger society, Brussels
Fertig, M./Kluve, J./Scheuer, M. (2005): Was hat die Reform der Minijobs bewirkt? Erfahrungen nach einem Jahr, RWI-Schriften 77, Berlin Heilemann, U./Ulrich, J. (2008): Viel Lärm um wenig? Zur Empirie von Lohnformeln in der Bundesrepublik, Wirtschaftswissenschaftliche Fakultät der Universität Leipzig, Diskussionspapier 68, Leipzig

Kettner, A./Rebien, M. (2007): Hartz-IV-Reform: Impulse für den Arbeitsmarkt, IAB-Kurzbericht 19, Nürnberg

Kronauer, M./Vogel, B. (1993): Erfahrungen mit Arbeitslosigkeit heute: Zwischen Chance auf Zeit und sozialer Ausgrenzung, SOFI-Mitteilungen 20, S. 1-14

Layard, R./Nickell, S./Jackman, R. (2005): Unemployment: Macroeconomic Performance and the Labour Market, second edition, Oxford OECD (1999): How do part-time jobs compare with full-time jobs?, OECD Employment Outlook, S. 18-46 Promberger, M. (2006): Leiharbeit im Betrieb. Strukturen, Kontexte und Handhabung einer atypischen Beschäftigungsform, Nürnberg Rudolph, H. (1999): Geringfügige Beschäftigung. Das 630-DM-Gesetz: Was ändert sich für wen?, IAB-Kurzbericht 11, Nürnberg

Rudolph, H. (2003): Mini- und Midi-Jobs. Geringfügige Beschäftigung im neuen Outfit, IAB-Kurzbericht 6, Nürnberg Wanger, S. (2006): Arbeitszeitpolitik: Teilzeitarbeit fördert Flexibilität und Produktivität, IAB-Kurzbericht 7, Nürnberg 